\title{
THE ROLE OF FOURIER MODES IN EXTENSION THEOREMS OF HARTOGS-CHIRKA TYPE
}

\author{
DAVID E. BARRETT AND GAUTAM BHARALI
}

\begin{abstract}
We generalize Chirka's theorem on the extension of functions holomorphic in a neighbourhood of $\Gamma(F) \cup(\partial D \times D)$ - where $D$ is the open unit disc and $\Gamma(F)$ is the graph of a continuous $D$-valued function $F$ - to the bidisc. We extend holomorphic functions by applying the Kontinuitätssatz to certain continuous families of analytic annuli, which is a procedure suited to configurations not covered by Chirka's theorem.
\end{abstract}

\section{Introduction AND STATEMENT OF RESUlts}

This article is motivated by the paper [3] by Chirka, in which the following theorem is proved (in what follows, $D$ will represent the open unit disc in $\mathbb{C}$ with centre at the origin, and given a function $F$ defined in some region in $\mathbb{C}, \Gamma(F)$ will denote the graph of $F$ over its domain) :

Theorem (Chirka). Let $F \in \mathcal{C}(\bar{D} ; \mathbb{C})$ and assume that $\sup _{\bar{D}}|F|<1$. Let $\Omega$ be a connected neighbourhood of $\Gamma(F) \cup(\partial D \times D)$ contained in $\mathbb{C} \times D$. If $f \in \mathcal{O}(\Omega)$, then $f$ extends holomorphically to the bidisc $D \times D$.

The requirement that $\sup _{\bar{D}}|F|<1$ is rather essential to the extension theorem stated above (in contrast, refer to [4] for a version by Chirka \& Rosay, in which the condition $\sup _{\bar{D}}|F|<1$ is relaxed, but in which only the functions holomorphic in the union of a neighbourhood of $\Gamma(F)$ with $\{z \in \mathbb{C}:|z|>1\} \times D$ - i.e. holomorphic in a large domain - extend holomorphically). A pertinent counterexample, when $\sup _{\bar{D}}|F|>1$, to the sort of holomorphic extension described in Chirka's theorem - i.e. extension from small neighbourhoods of $\Gamma(F) \cup(\partial D \times D)$ - is the case when $\Gamma(F)$ is a Wermer disc. We will discuss this example in $\$$ below.

The strategy of Chirka - inspired by the methods in [7] - is to construct a continuously varying family of functions $\left\{F_{t}\right\}_{t \in[0,1]} \subset\left\{G \in \mathcal{C}(\mathbb{C}) \mid \lim _{|z| \rightarrow \infty} G(z)=0\right\}$ such that $F_{1}=\widetilde{F}$ and $F_{0} \equiv 0$, and such that $\Gamma\left(F_{t}\right)$ is complex-analytic in a neighbourhood of any $\left(z, F_{t}(z)\right) \notin \Omega \cup(\{|z|>1\} \times D)$. Here, $\widetilde{F}$ is any smooth extension of the $F$ provided by the theorem, that satisfies $\left.\widetilde{F}\right|_{|z| \geq 2} \equiv 0$. Next, one extends $f \in \mathcal{O}(\Omega)$ to $\Omega \cup(\{|z|>1\} \times D)$ via Laurent decomposition. One can now show that the latter can be analytically continued, owing to the Kontinuitätssatz, via $\left\{F_{t}\right\}_{t \in[0,1]}$ to a neighbourhood of the classical Hartogs configuration $\Gamma\left(F_{0}\right) \cup(\partial D \times D)$. The condition $\sup _{\bar{D}}|F|<1$ is crucial in ensuring that the extension of $f \in \mathcal{O}(\Omega)$ by Laurent decomposition is single-valued. The strategy described fails in $\mathbb{C}^{n}, n>2$, and Chirka's theorem does not extend to higher dimensions as shown by Rosay's counterexample in [8].

The results in this paper are motivated by the two-fold aim of :

2000 Mathematics Subject Classification. Primary 32D15.

Key words and phrases. Hartogs-Chirka type extension, holomorphic extension.

The work of the first author is supported by NSF Grant DMS-0072237. 
a) Showing that functions holomorphic in small neighbourhoods of a Hartogs-Chirka type configuration $\Gamma(F) \cup(\partial D \times D)$, with $\sup _{\bar{D}}|F| \gg 1$, extend holomorphically to $D \times D$ (in a manner that will be made precise in Theorem 1.1), given that $F$ satisfies suitable restrictions.

b) Extending Chirka's theorem to higher dimensions, and to a reasonably wide class of Hartogs-Chirka type configurations $\Gamma(F) \cup\left(\partial D \times D^{m}\right), m \geq 2$ (in particular, to configurations in which $F$ is not merely real-analytic or $\left.\mathcal{C}^{\infty}\right)$.

Neither of the above seems to be achievable using Chirka's strategy. In this article, we discuss an alternative strategy for invoking the Kontinuitätssatz, and use it to demonstrate new HartogsChirka type extension phenomena.

The first of the above aims is met by the following theorem. But we first present the following notation : if $\Omega$ is a domain in $\mathbb{C}^{n}$, then $\left(\widetilde{\Omega}, \pi^{\Omega}\right)$ will denote the envelope of holomorphy of $\Omega$.

Theorem 1.1. Let $F \in \mathcal{C}(\bar{D} ; \mathbb{C})$ and assume that $\sup _{\partial D}|F|<1$. Let $A_{j}(r)$ represent the $j^{\text {th }}$ Fourier coefficient of $F\left(r e^{i \cdot}\right), r>0, j \in \mathbb{Z}$. Assume that $F$ satisfies the condition

$$
\sum_{n \in \mathbb{Z}} \frac{\left|A_{n}(r)\right|}{r^{n}}<1 \forall r \in(0,1] .
$$

Let $\Omega_{1}$ be a neighbourhood of $\Gamma(F) \cup(\partial D \times D)$ and let $\Omega_{2}$ be any connected open set satisfying $\partial D \times D \subset \overline{\Omega_{2}} \subset \Omega_{1} \cap(\{|z| \geq 1\} \times D)$. If $f \in \mathcal{O}\left(\Omega_{1}\right)$, then $\left.f\right|_{\Omega_{2}}$ has a holomorphic extension to $D \times D$.

Note that since $\sup _{\bar{D}}|F|>1, \pi^{\Omega_{1}}\left(\widetilde{\Omega_{1}}\right) \nsupseteq D \times D$ in general. For this reason, the usual arguments justifying that $f$ has a single-valued extension to the bidisc fail. This is the reason behind the particular form of the conclusion of Theorem 1.1 Observe that while the condition (1.1) admits $F$ such that the negative Fourier modes of $F\left(r e^{i \cdot}\right)$ are large, it imposes a severe restriction on the sizes of the positive Fourier modes of $F\left(r e^{i \cdot}\right)$ as $r \rightarrow 0^{+}$. One would like to investigate if such severe restrictions on the positive Fourier modes are necessary. This is a valid concern because if we assume that the function $F$ has only positive Fourier modes, the condition (1.1) becomes unnecessary. The relevant theorem in this case is

Theorem 1.2. Let $F \in \mathcal{C}(\bar{D} ; \mathbb{C})$ and assume that $\sup _{\partial D}|F|<1$. Let $A_{j}(r)$ represent the $j^{\text {th }}$ Fourier coefficient of $F\left(r e^{i \cdot}\right), r>0, j \in \mathbb{Z}$. Assume that $A_{j} \equiv 0 \forall j<0$. Let $\Omega_{1}$ be a neighbourhood of $\Gamma(F) \cup(\partial D \times D)$ and let $\Omega_{2}$ be any connected open set satisfying $\partial D \times D \subset \overline{\Omega_{2}} \subset \Omega_{1} \cap(\{|z| \geq$ $1\} \times D)$. If $f \in \mathcal{O}\left(\Omega_{1}\right)$, then $\left.f\right|_{\Omega_{2}}$ has a holomorphic extension to $D \times D$.

In a somewhat different direction, we may consider a continuous mapping $F:=\left(F_{1}, \ldots, F_{m}\right)$ : $\bar{D} \rightarrow D^{m}, m \geq 2$, and consider the Hartogs-Chirka type configuration $\Gamma(F) \cup\left(\partial D \times D^{m}\right)$. We know that, in general, Chirka's result is not true for such higher-dimensional configurations - see 8 . In contrast, it has been shown by Bharali [2] that Chirka's result does generalize to a certain class of Hartogs-Chirka type configurations. However, the class of real-analytic maps $\left(F_{1}, \ldots, F_{m}\right)$ studied in [2] is rather restrictive. We show in this paper that that if we impose a condition analogous to condition (1.1) above, we can demonstrate analytic continuation for a considerably less restrictive set of configurations. We make this precise in the following 
Theorem 1.3. Let $F=\left(F_{1}, \ldots, F_{m}\right) \in \mathcal{C}\left(\bar{D} ; \mathbb{C}^{m}\right)$. Assume that $F\left(e^{i \theta}\right) \in D^{m} \forall \theta \in[0,2 \pi)$ and let $A_{j k}(r)$ represent the $k^{\text {th }}$ Fourier coefficient of $F_{j}\left(r e^{i \cdot}\right), r>0, k \in \mathbb{Z}, j=1, \ldots, m$. Assume that each $F_{j}$ satisfies the condition

$$
\sum_{n \in \mathbb{Z}} \frac{\left|A_{j n}(r)\right|}{r^{n}}<1 \forall r \in(0,1] .
$$

Let $\Omega_{1}$ be a neighbourhood of $\Gamma(F) \cup\left(\partial D \times D^{m}\right)$ and let $\Omega_{2}$ be any connected open set satisfying $\partial D \times D^{m} \subset \overline{\Omega_{2}} \subset \Omega_{1} \cap\left(\{|z| \geq 1\} \times D^{m}\right)$. If $f \in \mathcal{O}\left(\Omega_{1}\right)$, then $\left.f\right|_{\Omega_{2}}$ has a holomorphic extension to $D \times D$.

We note that if, in Theorem 1.3 . $F$ were to satisfy the restriction $F(\zeta) \in D^{m} \forall \zeta \in \bar{D}$, then all functions $f \in \mathcal{O}(\Omega)$ - where $\Omega$ is a connected neighbourhood of $\Gamma(F) \cup\left(\partial D \times D^{m}\right)$ contained in $\mathbb{C} \times D^{m}$ - would extend to $D^{m}$, which is just Chirka's extension phenomenon in a restricted, higher-dimensional setting.

The approach used in the first and the third theorem is to construct a continuous family of analytic annuli which are attached to $\Gamma(H)$ - where $H$ is an appropriately selected perturbation of $F$ - along their inner boundaries, and to $\partial D \times D^{m}$ (with $m=1$ in Theorem 1.1 and $m \geq 2$ in Theorem 1.31) along their outer boundaries. Once this family is constructed, analytic continuation is achieved by invoking the Kontinuitätssatz. The proof of Theorem 1.2 uses a similar idea, but involves continuous families of analytic discs. These proofs may be found in 3 The technical construction of the aforementioned families of annuli/discs is carried out in the next section.

In the final section of this paper, we discuss a few examples. Firstly, we show that one can construct Hartogs-Chirka type configurations $\Gamma(F) \cup(\partial D \times D)$ such that $\sup _{\bar{D}}|F|$ is as large as we want and such that functions holomorphic in small neighbourhoods of this configuration extend. Next, we discuss a configuration involving Wermer's disc (see Example 4.2 for a definition) - for which the extension phenomenon occuring in the previous example fails. And lastly, we show how Rosay's counterexample to a higher-dimensional analogue of Chirka's theorem fails to satisfy the hypotheses of Theorem 1.3 .

\section{Preliminary lemmas}

We need a few preliminary lemmas before we can prove our main theorems. In what follows, $\operatorname{Ann}(a ; r, R)$ will denote the open annulus with centre at $a \in \mathbb{C}$ and having inner and outer radii $r$ and $R$ respectively, while $D(a ; R)$ will denote the open disc of radius $R$ with centre at $a$. The symbol $\mathcal{C}^{\infty}\left(\bar{D} ; \mathbb{C}^{m}\right), m=1,2, \ldots$, will denote the class of infinitely differentiable functions on the unit disc, all of whose derivatives extend to continuous functions on $\bar{D}$.

The reader will notice that in the following lemma the hypothesis $G \in \mathcal{C}^{\infty}(\bar{D} ; \mathbb{C})$ is much stronger than is required for the conclusion of Lemma 2.1. The only place where we use this hypothesis is in showing the existence of a certain limit towards the end of the proof. However, stating the strongest versions of Lemmas 2.1] and 2.3 - which are of relatively minimal utility in themselves merely results in statements that are overly technical. For this reason, the $G$ occuring in Lemmas 2.12.4 shall be assumed to be $\mathcal{C}^{\infty}$. 
Lemma 2.1. Let $G\left(r e^{i \theta}\right)=\sum_{n=-N}^{N} b_{n}(r) e^{i n \theta}$ and assume that $G \in \mathcal{C}^{\infty}(\bar{D} ; \mathbb{C})$. Assume further that

$$
\sum_{n=-N}^{N} \frac{\left|b_{n}(r)\right|}{r^{n}}<1 \forall r \in(0,1]
$$

Then the holomorphic function

$$
\mathcal{A}_{r}(\zeta)=\sum_{n=-N}^{N} b_{n}(r)\left(\frac{\zeta}{r}\right)^{n}, \quad \zeta \in \operatorname{Ann}(0 ; r, 1),
$$

which belongs to $\mathcal{O}[\operatorname{Ann}(0 ; r, 1)] \cap \mathcal{C}[\overline{\operatorname{Ann}}(0 ; r, 1)]$, satisfies $\left|\mathcal{A}_{r}\left(e^{i \theta}\right)\right|<1$. Fix $\nu \in \mathbb{N}$ and let $K \Subset$ $\operatorname{Ann}(0 ; 1 / \nu, 1)$ be a compact subset. The function $(0,1 / \nu] \times K \ni(r, \zeta) \mapsto \mathcal{A}_{r}(\zeta)$ extends to a continuous function on $[0,1 / \nu] \times K$.

Proof. To prove the first part of this lemma, note that

$$
\left|\mathcal{A}_{r}\left(e^{i \theta}\right)\right| \leq \sum_{n=-N}^{N}\left|b_{n}(r)\right|\left|\frac{e^{i \theta}}{r}\right|^{n}=\sum_{n=-N}^{N} \frac{\left|b_{n}(r)\right|}{r^{n}}<1 .
$$

We fix $\nu \in \mathbb{N}$ and then fix a compact set $K \Subset \operatorname{Ann}(0 ; 1 / \nu, 1)$. It is obvious that $(0, \nu] \times K \ni(r, \zeta) \mapsto$ $b_{-n}(r)(r / \zeta)^{n}$ extends to a continuous function on $[0,1 / \nu] \times K$, which simply vanishes when $r=0$, for each $n=1,2, \ldots, N$. Now consider the function $(r, \zeta) \mapsto b_{n}(r)(\zeta / r)^{n}, n=1,2, \ldots, N$. Note that (2.1) $\Longrightarrow\left|b_{n}(r)\right|<r^{n} \forall n=1,2, \ldots, N$. This implies, since $G$ is assumed to be smooth, that each of the latter functions extends continuously to a function $\varphi_{n} \in \mathcal{C}([0,1 / \nu] \times K)$, which is defined as

$$
\varphi_{n}(r, \zeta):= \begin{cases}b_{n}(r)(\zeta / r)^{n}, & \text { if }(r, \zeta) \in(0,1 / \nu] \times K, \\ \left.\frac{1}{n !} \frac{d^{n} b_{n}}{d r^{n}}\right|_{r=0} \zeta^{n}, & \text { if }(r, \zeta) \in\{0\} \times K .\end{cases}
$$

Since $\mathcal{A}_{r}$ is a finite sum of the functions $b_{n}(r)(r / \zeta)^{n}$, the last two observations establish the second part of this lemma.

Lemma 2.2. Let $G$ be as in Lemma [2.1, but assume additionally that $\sup _{\partial D}|G|<1$. Let $\Omega_{1}$ be a neighbourhood of $\Gamma(G) \cup(\partial D \times D)$ such as that described in Theorem 1.1. Then

a) $\left\{\mathcal{A}_{r}\right\}_{r \in(0,1)}$ is a continuous family in the sense that for a fixed $\zeta_{0} \in D \backslash\{0\}, r \mapsto \mathcal{A}_{r}\left(\zeta_{0}\right)$ is continuous in the interval $\left(0,\left|\zeta_{0}\right|\right)$.

b) $\lim _{r \rightarrow 0^{+}} \mathcal{A}_{r}\left(\zeta_{0}\right)$ exists for each $\zeta_{0} \in D \backslash\{0\}$, and there exists a $\psi \in \mathcal{O}(D)$ such that $\psi(\zeta)=\lim _{r \rightarrow 0^{+}} \mathcal{A}_{r}(\zeta)$ on $D \backslash\{0\}$.

c) Define

$$
\mathfrak{K}:=\Gamma(\psi) \cup\left[\cup_{0<r<1}\left\{\left(\zeta, \mathcal{A}_{r}(\zeta)\right) \in \mathbb{C}^{2}|r<| \zeta \mid<1\right\}\right] \backslash \Omega_{1} .
$$

$\mathfrak{K}$ is compact.

Proof. Part (a) and the first half of part (b) are obvious conclusions of Lemma 2.1 Thus, we may define

$$
\psi(\zeta):=\lim _{|\zeta|>r \rightarrow 0^{+}} \mathcal{A}_{r}(\zeta) \quad \forall \zeta \in D \backslash\{0\} .
$$

Fix a $\nu \in \mathbb{N}$. Lemma 2.1 (b) tells us that

$$
\left(\left.\mathcal{A}_{r}\right|_{\operatorname{Ann}(0 ; 1 / \nu, 1)}\right)(\zeta) \longrightarrow \psi(\zeta) \text { uniformly on each compact } K \Subset \operatorname{Ann}(0 ; 1 / \nu, 1) \text { as } r \searrow 0 \text {. }
$$


We conclude from this statement that

$$
\left.\psi\right|_{\text {Ann }(0 ; 1 / \nu, 1)} \in \mathcal{O}[\operatorname{Ann}(0 ; 1 / \nu, 1)] \quad \forall \nu=2,3,4, \ldots
$$

Before proceeding any further, we comment that the functions $\mathcal{A}_{r}$ are so constructed that $\Gamma\left(\mathcal{A}_{r}\right)$, $0<r<1$, are analytic annuli that are attached to $\Gamma(G)$ along their inner boundaries and - in view of the inequality 2.2 - to $\partial D \times D$ along their outer boundaries. Therefore,

$$
\begin{aligned}
\left|\mathcal{A}_{r}(\zeta)\right| \leq \max \left\{\sup _{|\xi|=r}\left|\mathcal{A}_{r}(\xi)\right|, 1\right\} \leq \max \left\{\begin{array}{l}
\left.\sup _{\bar{D}}|G|, 1\right\} \\
\forall \zeta \in \operatorname{Ann}(0 ; r, 1) \text { and for each } r \in(0,1) .
\end{array}\right.
\end{aligned}
$$

By (2.3), $\psi$ is already holomorphic on $D \backslash\{0\}$. The bounds above imply, since $\psi(\zeta)$ is the limit of the $\mathcal{A}_{r}(\zeta)$ 's, provided $\zeta \neq 0$, that $|\psi(\zeta)| \leq \sup _{\xi \neq 0}|G(\xi)|$ in a punctured neighbourhood of the origin. Thus, $\psi$ extends to a holomorphic function on $D$. This establishes (b).

Notice that by the estimates (2.4) and part (b) of this lemma, $\mathfrak{K}$ is a bounded set. Therefore, it suffices to show that $\mathfrak{K}$ is closed. Now consider a point $(z, w) \notin \Omega_{1}$ with the property that there exist sequences $\{r(\nu)\}_{\nu \in \mathbb{N}} \subset(0,1)$ and $\left\{\zeta_{\nu}\right\}_{\nu \in \mathbb{N}} \subset D$ such that $r(\nu) \rightarrow 0$ as $\nu \rightarrow \infty$ and $\left(\zeta_{\nu}, \mathcal{A}_{r(\nu)}\left(\zeta_{\nu}\right)\right) \longrightarrow(z, w)$ as $\nu \rightarrow \infty$. It is easy to see that to prove (c), it suffices to show that all such points $(z, w) \in \mathfrak{K}$. Notice that, by construction, there is a $\delta\left(\Omega_{1}\right)>0$ depending only on $\Omega_{1}$ such that $\left(\zeta, \mathcal{A}_{r}(\zeta)\right) \in \Omega_{1} \forall r,|\zeta|<\delta\left(\Omega_{1}\right)$. Thus, as $(z, w) \notin \Omega_{1}, z \neq 0$. Now, given that the $\mathcal{A}_{r}$ 's converge uniformly on compact subsets lying away from 0 , there exists $\kappa_{1} \in \mathbb{N}$ such that

$$
\left|\mathcal{A}_{r(\nu)}(\zeta)-\psi(\zeta)\right|<\varepsilon / 2 \quad \forall \nu \geq \kappa_{1}, \forall \zeta \in \bar{D}(z ;|z| / 2)
$$

Let $\kappa_{2} \in \mathbb{N}$ be such that

$$
\zeta_{\nu} \in D(z ;|z| / 2) \quad \text { and } \quad\left|w-\mathcal{A}_{r(\nu)}\left(\zeta_{\nu}\right)\right|<\varepsilon / 2 \quad \forall \nu \geq \kappa_{2} .
$$

The above inequalities imply that

$$
\left|w-\psi\left(\zeta_{\nu}\right)\right| \leq\left|w-\mathcal{A}_{r(\nu)}\left(\zeta_{\nu}\right)\right|+\left|\mathcal{A}_{r(\nu)}\left(\zeta_{\nu}\right)-\psi\left(\zeta_{\nu}\right)\right|<\varepsilon \quad \forall \nu \geq \max \left(\kappa_{1}, \kappa_{2}\right) .
$$

This tells us that $w=\lim _{\nu \rightarrow \infty} \psi\left(\zeta_{\nu}\right)$, whence $(z, w) \in \Gamma(\psi) \backslash \Omega_{1}$. This establishes (c), and concludes our proof.

Lemma 2.3. Let $G\left(r e^{i \theta}\right)=\sum_{n=0}^{N} b_{n}(r) e^{i n \theta}-i . e$. we assume that $G\left(r e^{i \cdot}\right)$ has no negative Fourier modes. Assume further that $G \in \mathcal{C}^{\infty}(\bar{D} ; \mathbb{C})$. Then the holomorphic function

$$
\mathcal{D}_{r}(\zeta)=\sum_{n=0}^{N} b_{n}(r)\left(\frac{\zeta}{r}\right)^{n}, \quad \zeta \in D(0 ; r),
$$

which belongs to $\mathcal{O}[D(0 ; r)] \cap \mathcal{C}[\bar{D}(0 ; r)]$, satisfies $\mathcal{D}_{r}\left(r e^{i \theta}\right)=G\left(r e^{i \theta}\right) \forall \theta \in[0,2 \pi)$. Fix $\nu \in \mathbb{N}$ and let $K \Subset D(0 ; 1-1 / \nu)$ be a compact subset. The function $(r, \zeta) \mapsto \mathcal{D}_{r}(\zeta)$ is a continuous function on $[1-1 / \nu, 1] \times K$.

The above lemma is a triviality; we merely state it as an element that will be needed in the proof of our next result.

Lemma 2.4. Let $G$ be as in Lemma 2.3] but assume additionally that $\sup _{\partial D}|G|<1$. Let $\Omega_{1}$ be a neighbourhood of $\Gamma(G) \cup(\partial D \times D)$ such as that described in Theorem 1.2. Then 
a) $\left\{\mathcal{D}_{r}\right\}_{r \in(0,1)}$ is a continuous family in the sense that for a fixed $\zeta_{0} \in D, r \mapsto \mathcal{D}_{r}\left(\zeta_{0}\right)$ is continuous in the interval $\left(\left|\zeta_{0}\right|, 1\right)$.

b) $\lim _{r \rightarrow 1^{-}} \mathcal{D}_{r}(\zeta)$ exists for each $\zeta \in D$, and this limit defines a holomorphic function $\psi \in$ $\mathcal{O}(D)$.

c) Define

$$
\mathfrak{K}:=\Gamma(\psi) \cup\left[\cup_{0<r<1}\left\{\left(\zeta, \mathcal{D}_{r}(\zeta)\right) \in \mathbb{C}^{2}|| \zeta \mid<r\right\}\right] \backslash \Omega_{1} .
$$

$\mathfrak{K}$ is compact.

Proof. Part (a) and the first half of part (b) are direct consequences of Lemma 2.3. The inference that

$$
\psi(\zeta):=\lim _{|\zeta|<r \rightarrow 1^{-}} \mathcal{D}_{r}(\zeta) \quad \forall \zeta \in D
$$

is holomorphic follows from Lemma 2.3 The uniform-convergence argument is exactly analogous to the argument used in proving Lemma 2.2 We therefore omit the details. We remark that

$$
\psi(\zeta)=\sum_{n=0}^{N} b_{n}(1) \zeta^{n} .
$$

The functions $\mathcal{D}_{r}$ are so constructed that $\Gamma\left(\mathcal{D}_{r}\right), 0<r<1$, are analytic discs that are attached to $\Gamma(G)$ along their boundaries. Therefore,

$$
\left|\mathcal{D}_{r}(\zeta)\right| \leq \sup _{|\xi|=r}\left|\mathcal{D}_{r}(\xi)\right| \leq \sup _{\bar{D}}|G| \quad \forall \zeta \in D(0 ; r) \text { and for each } r \geq 1-1 / \nu .
$$

Thus, $\mathfrak{K}$ is a bounded set, and we argue that $\mathfrak{K}$ is closed exactly as we did in Lemma 2.2 (c).

The following lemma is key to the proofs of Theorems 1.11.3 Before proving it, we explicitly state the following simple

Fact : Due to the continuity of the functions $F$ and $G$ occuring in the statements of the various theorems and lemmas above, the associated Fourier coefficients $A_{n}(r)$ and $b_{n}(r)$ satisfy $A_{n}(0)=$ $b_{n}(0)=0 \forall n \neq 0$.

This fact is used implicitly at several places in the next lemma.

Lemma 2.5. Let $F \in \mathcal{C}(\bar{D} ; \mathbb{C})$ and let $A_{j}(r)$ represent the $j^{\text {th }}$ Fourier coefficient of $F\left(r e^{i \cdot}\right)$, $r>0, j \in \mathbb{Z}$. Assume that :

1) $\sup _{\partial D}|F|<1$, and

2) $F$ satisfies the condition

$$
\sum_{n \in \mathbb{Z}} \frac{\left|A_{n}(r)\right|}{r^{n}}<1 \quad \forall r \in(0,1] .
$$

Given $\varepsilon>0$ there exists a function $G \in \mathcal{C}^{\infty}(\bar{D} ; \mathbb{C})$ of the form

$$
G\left(r e^{i \theta}\right)=\sum_{n=-N}^{N} B_{n}(r) e^{i n \theta},
$$

where $N$ is some large positive integer and $B_{n} \in \mathcal{C}^{\infty}([0,1] ; \mathbb{C})$, such that

- $|F(\zeta)-G(\zeta)|<\varepsilon \forall \zeta \in \bar{D}$,

- $G$ has the property (1) and satisfies the analogue of (2) above (with $B_{n}(r)$ replacing $A_{n}(r)$ in (2.5) above). 
Furthermore, if property (2) is replaced by

$\left.2^{*}\right) F$ has no negative Fourier modes,

then $G$ can be constructed so that it has property (1) and $B_{-j} \equiv 0$ for $j=1,2, \ldots, N$.

Proof. Define

$$
\begin{aligned}
S_{m}(\theta, r) & :=\sum_{j=-m}^{m} A_{j}(r) e^{i j \theta} \\
\sigma_{n}(\theta, r) & :=\frac{S_{0}(r, \theta)+\cdots+S_{n}(\theta, r)}{n+1}
\end{aligned}
$$

Let us first assume that $F$ has properties (1) and (2). Let $\eta>0$ be so small that

$$
\begin{aligned}
\eta & <1-\sup _{\partial D}|F|, \\
\eta+\sum_{n \in \mathbb{Z}}\left|A_{n}(r)\right| / r^{n} & <1 \quad \forall r \in(0 ; 1],
\end{aligned}
$$

and define $\delta:=\min (\varepsilon, \eta)$. There exists a natural number $N>0$ such that

$$
\left|F\left(r e^{i \theta}\right)-\sigma_{N}(\theta, r)\right|<\delta / 2 \quad \forall(\theta, r) \in[0,2 \pi) \times[0,1] .
$$

This above is a consequence of Fejér's theorem. For a fixed $r \in[0,1]$, (2.7) is precisely the statement of Fejér's theorem applied to the periodic function $F\left(r e^{i \cdot}\right)$. However, on examining the proof of Fejér's theorem, one sees that owing to the equicontinuity of the family $\left\{F\left(r e^{i \cdot}\right)\right\}_{r \in[0,1]} \subset \mathcal{C}(\mathbb{T})$, the choice of $N$ in (2.7) is uniform in $r \in[0,1]$.

One sees immediately that if one writes

$$
\sigma_{N}(\theta, r)=\sum_{j=-N}^{N} a_{j}(r) e^{i j \theta},
$$

then $\left|a_{j}(r)\right| \leq\left|A_{j}(r)\right| \forall r \in[0,1]$. For each $j=1,2, \ldots, N$, we pick a function $B_{-j}(r)$ which satisfies the following conditions :

i) $B_{-j} \in \mathcal{C}^{\infty}([0,1] ; \mathbb{C})$,

ii) $B_{-j}$ vanishes to infinite order at $r=0$, and

iii) $\left|a_{-j}(r)-B_{-j}(r)\right| \leq \delta / 2(2 N+1) \forall r \in[0,1]$,

provided $a_{-j} \not \equiv 0, j=1,2, \ldots, N$. If $a_{-j} \equiv 0$, we just choose $B_{-j} \equiv 0$. Note that by our condition on the Fourier coefficients $\left\{A_{n}(r)\right\}_{n \in \mathbb{Z}},\left|a_{j}(r)\right| \leq\left|A_{j}(r)\right| \leq r^{j} \forall j=1,2, \ldots, N, r \in[0,1)$. Let $0<R_{0}<1$ be a small number such that

$$
R_{0} \leq\left\{\frac{\delta}{4(2 N+1)}\right\}^{1 / j} \quad \forall j=1,2, \ldots, N .
$$

For each $j=1,2, \ldots, N$ we define a function $B_{j}(r)$ as follows :

$$
B_{j}(r):= \begin{cases}\alpha_{j}(r) r^{j}, & \text { if } r \leq R_{0}, \\ \beta_{j}(r), & \text { if } r \geq R_{0},\end{cases}
$$

such that

$\left.i^{*}\right) B_{j} \in \mathcal{C}^{\infty}([0,1] ; \mathbb{C})$

$\left.i i^{*}\right) \alpha_{j}$ vanishes to infinite order at $r=0$, 
$\left.i i i^{*}\right) \alpha_{j}$ satisfies

$$
\left|\alpha_{j}(r)\right| \leq \sup _{s \leq 1} \frac{\left|a_{j}(s)\right|}{s^{j}} \quad \forall s \in\left[0, R_{0}\right]
$$

$\left.i v^{*}\right) \beta_{j}$ satisfies

$$
\left|\beta_{j}(r)-a_{j}(r)\right| \leq \frac{R_{0}^{j} \delta}{2(2 N+1)} \quad \forall r \in\left[R_{0}, 1\right]
$$

Finally, define $B_{0}(r)$ to be any $\mathcal{C}^{\infty}$ function such that $\left|B_{0}(r)-a_{0}(r)\right|<\delta / 2(2 N+1) \forall r \in[0,1]$ and such that $B_{0}-B_{0}(0)$ vanishes to high order at $r=0$. Now write

$$
G\left(r e^{i \theta}\right)=\sum_{j=-N}^{N} B_{j}(r) e^{i j \theta} .
$$

We now make some estimates. We first consider the $B_{-j}(r)$ 's, $j=1,2, \ldots, N$. Note that the following statements continue to be true trivially if $B_{-j} \equiv 0$ for any $j=1,2, \ldots, N$.

$$
\begin{aligned}
\sum_{j=1}^{N}\left|B_{-j}(r)-a_{-j}(r)\right| & \leq \sum_{j=1}^{N} \frac{\delta}{2(2 N+1)} \leq \frac{\delta N}{2(2 N+1)}, \\
\sum_{j=1}^{N}\left|B_{-j}(r)\right| r^{j} & \leq \sum_{j=1}^{N} r^{j}\left\{\left|a_{-j}(r)\right|+\frac{\delta}{2(2 N+1)}\right\} \\
& \leq \sum_{j=1}^{N}\left|a_{-j}(r)\right| r^{j}+\frac{\delta N}{2(2 N+1)} \quad \forall r \in[0,1] .
\end{aligned}
$$

Next, we consider the $B_{j}(r)$ 's, $j=1,2, \ldots, N$. First, we let $0 \leq r \leq R_{0}$. We use item $\left(i i i^{*}\right)$ in the definition of $B_{j}(r)$ above to get :

$$
\begin{aligned}
\sum_{j=1}^{N}\left|B_{j}(r)-a_{j}(r)\right| & \leq \sum_{j=1}^{N} r^{j}\left|\alpha_{j}(r)-\frac{\left|a_{j}(r)\right|}{r^{j}}\right| \\
& \leq \sum_{j=1}^{N} 2 R_{0}^{j} \sup _{s \leq 1} \frac{\left|a_{j}(s)\right|}{s^{j}} \\
& \leq \sum_{j=1}^{N} 2\left\{\frac{\delta}{4(2 N+1)}\right\} \sup _{s \leq 1} \frac{\left|A_{j}(s)\right|}{s^{j}} \\
& \leq \frac{\delta N}{2(2 N+1)}, \\
\sum_{j=1}^{N} \frac{\left|B_{j}(r)\right|}{r^{j}} & \leq \sum_{j=1}^{N} \sup _{s \leq 1} \frac{\left|a_{j}(s)\right|}{s^{j}} \quad \forall r \in\left[0, R_{0}\right] .
\end{aligned}
$$


And when we consider $R_{0} \leq r \leq 1$, we use item $\left(i v^{*}\right)$ in the definition of $B_{j}(r)$ to get :

$$
\begin{aligned}
\sum_{j=1}^{N}\left|B_{j}(r)-a_{j}(r)\right| & \leq \sum_{j=1}^{N} \frac{R_{0}^{j} \delta}{2(2 N+1)} \leq \frac{\delta N}{2(2 N+1)}, \\
\sum_{j=1}^{N} \frac{\left|B_{j}(r)\right|}{r^{j}} & \leq \sum_{j=1}^{N}\left\{\frac{\left|a_{j}(r)\right|}{r^{j}}+\frac{R_{0}^{j} \delta}{2(2 N+1) R_{0}^{j}}\right\} \\
& \leq \sum_{j=1}^{N} \frac{\left|a_{j}(r)\right|}{r^{j}}+\frac{\delta N}{2(2 N+1)} \quad \forall r \in\left[R_{0}, 1\right] .
\end{aligned}
$$

Observe that from (2.9) and (2.11), we have

$$
\begin{aligned}
\sum_{j=-N}^{N} \frac{\left|B_{j}(r)\right|}{r^{j}} & \leq \sum_{j=1}^{N}\left|a_{-j}(r)\right| r^{j}+\frac{\delta N}{2(2 N+1)}+\left|a_{0}(r)\right|+\frac{\delta}{2(2 N+1)}+\sum_{j=1}^{N} \sup _{s \leq 1} \frac{\left|a_{j}(s)\right|}{s^{j}} \\
& \leq \sum_{j=-N}^{N} \sup _{s \leq 1} \frac{\left|a_{j}(s)\right|}{s^{j}}+\frac{\delta(N+1)}{2(2 N+1)} \\
& \leq \sum_{j=-N}^{N} \sup _{s \leq 1} \frac{\left|A_{j}(s)\right|}{s^{j}}+\frac{\eta}{2}<1 \quad \forall r \in\left[0, R_{0}\right] .
\end{aligned}
$$

The last inequality follows from the definition of $\eta$ and the fact that $\left|a_{j}(r)\right| \leq\left|A_{j}(r)\right| \forall r \in[0,1]$. Next, applying (2.9) and (2.13) we get

$$
\begin{aligned}
\sum_{j=-N}^{N} \frac{\left|B_{j}(r)\right|}{r^{j}} & \leq \sum_{j \neq 0} \frac{\left|a_{j}(r)\right|}{r^{j}}+\frac{2 \delta N}{2(2 N+1)}+\left|a_{0}(r)\right|+\frac{\delta}{2(2 N+1)} \\
& \leq \sum_{j=-N}^{N} \sup _{s \leq 1} \frac{\left|a_{j}(s)\right|}{s^{j}}+\frac{\delta}{2} \\
& \leq \sum_{j=-N}^{N} \sup _{s \leq 1} \frac{\left|A_{j}(s)\right|}{s^{j}}+\frac{\eta}{2}<1 \quad \forall r \in\left[R_{0}, 1\right] .
\end{aligned}
$$

From the inequalities (2.6), (2.14) and (2.15), we get

$$
\sum_{j=-N}^{N} \frac{\left|B_{j}(r)\right|}{r^{j}}<1 \quad \forall r \in[0,1],
$$

which is to say that $G$ satisfies the analogue of (2), with $B_{n}(r)$ replacing $A_{n}(r)$ in the expression (2.5).

We now exploit the estimates (2.8), (2.10) and (2.12), to get

$$
\begin{aligned}
\left|F\left(r e^{i \theta}\right)-G\left(r e^{i \theta}\right)\right| & \leq\left|F\left(r e^{i \theta}\right)-\sigma_{N}\left(r e^{i \theta}\right)\right|+\sum_{j=-N}^{N}\left|a_{j}(r)-B_{j}(r)\right| \\
& <\frac{\delta}{2}+2 \cdot \frac{\delta N}{2(2 N+1)}+\frac{\delta}{2(2 N+1)}=\delta .
\end{aligned}
$$

Given the way in which $\delta$ is defined, we see that $G$ has the property (1), and $|G(\zeta)-F(\zeta)|<$ $\varepsilon \forall \zeta \in \bar{D}$. $G$ is, of course, smooth by construction. 
Note further that in the above construction, if $F$ has no negative Fourier modes, neither does $G$. So, if $F$ had the property $\left(2^{*}\right)$ instead of property (2), in addition to choosing $B_{-j} \equiv 0$ we would use the same rule by which we selected $B_{0}(r)$ in the above argument to define $B_{j}(r), j=1,2, \ldots, N$. It is easy to verify that this modified construction would yield the second part of this lemma.

\section{Proofs of the theOREMS}

3.1. The proof of Theorem 1.1, Let $\varepsilon>0$ be so small that $F(D)+\eta \subset \Omega_{1} \forall \eta \in \mathbb{C}$ such that $|\eta|<2 \varepsilon$. By Lemma 2.5 there exists a function $H \in \mathcal{C}^{\infty}(\bar{D} ; \mathbb{C})$ with $H\left(r e^{i \theta}\right)=\sum_{n=-N}^{N} B_{n}(r) e^{i n \theta}$ such that

$$
\begin{array}{r}
|H(\zeta)-F(\zeta)|<\varepsilon \quad \forall \zeta \in \bar{D}, \quad \sup _{\partial D}|H|<1, \\
\sum_{n=-N}^{N} \frac{\left|B_{n}(r)\right|}{r^{n}}<1 \quad \forall r \in(0,1] .
\end{array}
$$

Let $\delta>0$ be so small that

- $\sup _{\partial D}|H|+\delta<1$

- $\delta+\sum_{n=-N}^{N}\left|B_{n}(r)\right| / r^{n}<1 \forall r \in(0 ; 1]$; and

- $H(D)+\eta \subset \Omega_{1} \forall \eta \in \mathbb{C}$ such that $|\eta|<\delta$.

Define, for each $\eta$ such that $|\eta|<\delta$

$$
\begin{aligned}
H^{(\eta)}(\zeta) & :=H(\zeta)+\eta, \\
\mathcal{A}_{r}^{(\eta)}(\zeta) & :=\sum_{n \neq 0} B_{n}(r)\left(\frac{\zeta}{r}\right)^{n}+\left(B_{0}(r)+\eta\right), \quad \zeta \in \operatorname{Ann}(0 ; r, 1),
\end{aligned}
$$

We apply Lemma 2.2 to $\left\{\mathcal{A}_{r}^{(\eta)}\right\}_{r \in(0 ; 1)}$ for each $\eta$, by taking

$$
b_{n}(r)=B_{n}(r) \quad \forall n \neq 0, \quad b_{0}(r)=B_{0}(r)+\eta
$$

in that lemma. We conclude that there is a function $\psi \in \mathcal{O}(D)$ such that

1) For any fixed $\zeta_{0} \in D \backslash\{0\}, r \mapsto \mathcal{A}_{r}^{(\eta)}\left(\zeta_{0}\right)$ is continuous in $\left(0,\left|\zeta_{0}\right|\right)$ for $|\eta|<\delta$.

2) For $\zeta \in D \backslash\{0\}, \lim _{r \rightarrow 0^{+}} \mathcal{A}_{r}^{(\eta)}(\zeta)=\psi(\zeta)+\eta$ for $|\eta|<\delta$.

3) For each $\eta:|\eta|<\delta, \mathfrak{K}^{(\eta)}$ is compact, where we define

$$
\mathfrak{K}^{(\eta)}:=\Gamma(\psi+\eta) \cup\left[\cup_{0<r<1}\left\{\left(\zeta, \mathcal{A}_{r}^{(\eta)}(\zeta)\right) \in \mathbb{C}^{2}|r<| \zeta \mid<1\right\}\right] \backslash \Omega_{1} .
$$

In other words, for each fixed $\eta$, the family $\left\{\Gamma\left(\mathcal{A}_{r}^{(\eta)}\right)\right\}_{r \in(0,1)}$ is a continuous family of analytic annuli attached to $\Gamma\left(H^{(\eta)}\right) \cup(\partial D \times D)$, which accumulate onto $\Gamma(\psi+\eta)$ as $r \rightarrow 0^{+}$. The analytic annuli $\Gamma\left(\mathcal{A}_{r}^{(\eta)}\right)$ with $r \approx 1$ are contained in $\Omega_{1}$. We can therefore apply the Kontinuitätssatz to conclude that

$$
U(\Delta):=\{(z, w) \in D \times \mathbb{C}:(w-\psi(z)) \in \Delta\}=\bigcup_{\eta \in \Delta} \Gamma(\psi+\eta) \subset \pi^{\Omega_{1}}\left(\widetilde{\Omega_{1}}\right),
$$

where $\Delta$ is any disc contained in $D(0 ; \delta)$

There is a canonical holomorphic imbedding of $\Omega_{1}$ into $\widetilde{\Omega_{1}}$. We denote this imbedding by $\mathrm{j}: \Omega_{1} \hookrightarrow \widetilde{\Omega_{1}}$. Corresponding to each $f \in \mathcal{O}\left(\Omega_{1}\right)$ there is a holomorphic function on $\widetilde{\Omega_{1}}$, which we 
shall denote by $\mathcal{E}(f)$, such that $\mathcal{E}(f) \circ \mathrm{j}=f$. It is now a standard argument - see, for instance [5] or [3] - to show that there exist holomorphic mappings

$$
\left.\widetilde{\mathcal{A}_{r}}(\cdot ; \eta): \operatorname{Ann}(0 ; r, 1) \rightarrow \widetilde{\Omega_{1}} \forall r \in(0,1) \text {, and } \widetilde{\psi(} \cdot ; \eta\right): D \rightarrow \widetilde{\Omega_{1}}
$$

such that

a) For each $\eta$ with $|\eta|<\delta$ :

$$
\begin{aligned}
\pi^{\Omega_{1}} \circ \widetilde{\mathcal{A}_{r}}(\zeta ; \eta) & =\left(\zeta, \mathcal{A}_{r}^{(\eta)}(\zeta)\right) \forall \zeta \in \operatorname{Ann}(0 ; r, 1) \text { when } r \in(0,1), \text { and } \\
\pi^{\Omega_{1}} \circ \widetilde{\psi}(\zeta ; \eta) & =(\zeta, \psi(\zeta)+\eta) \forall \zeta \in D .
\end{aligned}
$$

b) $\mathrm{j}\left(\zeta, \mathcal{A}_{r}^{(\eta)}(\zeta)\right)=\widetilde{\mathcal{A}_{r}}(\zeta ; \eta)$ wherever the left-hand side is defined, and $\forall r \in(0,1)$.

c) $\mathrm{j}(\zeta, \psi(\zeta)+\eta)=\widetilde{\psi}(\zeta ; \eta)$ wherever the left-hand side is defined.

Notice that - in view of item (a) - for each fixed $\zeta \in D, \eta \mapsto \widetilde{\psi}(\zeta ; \eta)$ is holomorphic.

Let $V$ be the connected component of $\left(\pi^{\Omega_{1}}\right)^{-1}(U(|\eta|<\delta))$ containing $\mathcal{C}_{0}:=\operatorname{image}(\widetilde{\psi}(\cdot ; 0))$. For each point $q \in \mathfrak{C}_{0}$ there is a neighbourhood $W(q) \Subset V$ of $q$ such that $\left.\pi^{\Omega_{1}}\right|_{W(q)}: W(q) \rightarrow \mathbb{C}^{2}$ is a biholomorphism. Let $\Delta_{*}$ be a disc centered at the origin that is so small that

$$
\operatorname{image}(\widetilde{\psi}(\cdot ; \eta)) \subset \bigcup_{q \in \mathfrak{C}_{0}} W(q) \quad \forall \eta \in \Delta_{*} .
$$

We define $\Omega^{*}:=U\left(\Delta_{*}\right) \cup \omega_{2}, \omega_{2}$ being a connected open set satisfying

- $\overline{\Omega_{2}} \subset \omega_{2} \subset \Omega_{1}$; and

- $\omega_{2} \cap U\left(\Delta_{*}\right)$ is connected,

where $\Omega_{2}$ is as described in Theorem 1.1 and $U\left(\Delta_{*}\right)$ is as defined by (3.1). Our goal is to map $\Omega^{*}$ into $\widetilde{\Omega_{1}}$ in such a way that this mapping extends $\mathrm{j}$. This mapping will allow us - given any $f \in \mathcal{O}\left(\Omega_{1}\right)$ - to extend $\left.f\right|_{\omega_{2}}$ to $\Omega^{*}$. But $\Omega^{*}$ is a neighbourhood of a classical Hartogs configuration, whence $\left.f\right|_{\Omega_{2}}$ would extend to the bidisc. To this end, we define

$$
\widetilde{\mathrm{j}}(z, w):= \begin{cases}\widetilde{\psi}(z ; w-\psi(z)), & \text { if }(z, w) \in U\left(\Delta_{*}\right), \\ \mathrm{j}(z, w), & \text { if }(z, w) \in \omega_{2} .\end{cases}
$$

Note that if $(z, w) \in U\left(\Delta_{*}\right) \cap \omega_{2}$, then, in view of item (c) above

$$
\widetilde{\psi}(z ; w-\psi(z))=\mathrm{j}(z, \psi(z)+\{w-\psi(z)\})=\mathrm{j}(z, w) \quad \forall(z, w) \in U\left(\Delta_{*}\right) \cap \omega_{2} .
$$

Thus, $\tilde{j}$ is well-defined, and extends j. From our foregoing remarks, $\tilde{j}$ is holomorphic. Given any $f \in \mathcal{O}\left(\Omega_{1}\right)$, we define $\widetilde{f} \in \mathcal{O}\left(\Omega_{*}\right)$ by $\tilde{f}:=\mathcal{E}(f) \circ \widetilde{j}$. In view of (3.2), $\left.\left.\widetilde{f}\right|_{\omega_{2}} \equiv f\right|_{\omega_{2}}$. Notice that $\Omega_{*}$ is a neighbourhood of $\Gamma(\psi) \cup(\partial D \times D)$, which is the classical Hartogs configuration. Thus $\tilde{f}$ has a holomorphic extension to $D \times D$, whence $\left.f\right|_{\Omega_{2}}$ has a holomorphic extension to $D \times D$. This concludes our proof.

3.2. The proof of Theorem 1.2, Since the proof of this theorem is similar to that of Theorem 1.1 we shall be brief. Let $\varepsilon>0$ be so small that $F(D)+\eta \subset \Omega_{1} \forall \eta \in \mathbb{C}$ such that $|\eta|<2 \varepsilon$. By Lemma 2.5 there exists a function $H \in \mathcal{C}^{\infty}(\bar{D} ; \mathbb{C})$ with $H\left(r e^{i \theta}\right)=\sum_{n=0}^{N} B_{n}(r) e^{i n \theta}$ such that

$$
|H(\zeta)-F(\zeta)|<\varepsilon \quad \forall \zeta \in \bar{D}, \quad \sup _{\partial D}|H|<1 .
$$


Note that the $H$ that has the two properties above can be so chosen that it has no negative Fourier modes. Let $\delta>0$ be so small that

- $\sup _{\partial D}|H|+\delta<1 ;$ and

- $H(D)+\eta \subset \Omega_{1} \forall \eta \in \mathbb{C}$ such that $|\eta|<\delta$.

For each $\eta$ such that $|\eta|<\delta$, we define

$$
\begin{aligned}
& H^{(\eta)}(\zeta):=H(\zeta)+\eta, \\
& \mathcal{D}_{r}^{(\eta)}(\zeta):=\sum_{n=1}^{N} B_{n}(r)\left(\frac{\zeta}{r}\right)^{n}+\left(B_{0}(r)+\eta\right), \quad \zeta \in D(0 ; r),
\end{aligned}
$$

We apply Lemma 2.4 to $\left\{\mathcal{D}_{r}^{(\eta)}\right\}_{r \in(0 ; 1)}$ for each $\eta$, by taking

$$
b_{n}(r)=B_{n}(r) \quad \forall n=1,2, \ldots, N, \quad b_{0}(r)=B_{0}(r)+\eta
$$

in that lemma. For each fixed $\eta$, the family $\left\{\Gamma\left(\mathcal{D}_{r}^{(\eta)}\right)\right\}_{r \in(0,1)}$ is a continuous family of analytic discs which are attached to $\Gamma\left(H^{(\eta)}\right)$ along their boundaries, and which accumulate onto a holomorphic graph $\Gamma(\psi+\eta)$ as $r \rightarrow 1^{-}$. Furthermore, for $|\eta|<\delta$, each

$$
\mathfrak{K}^{(\eta)}:=\Gamma(\psi+\eta) \cup\left[\cup_{0<r<1}\left\{\left(\zeta, \mathcal{D}_{r}^{(\eta)}(\zeta)\right) \in \mathbb{C}^{2}|| \zeta \mid<r\right\}\right] \backslash \Omega_{1}
$$

is compact. We may therefore apply the Kontinuitätssatz to conclude that

$$
U(\Delta):=\{(z, w) \in D \times \mathbb{C}:(w-\psi(z)) \in \Delta\}=\bigcup_{\eta \in \Delta} \Gamma(\psi+\eta) \subset \pi^{\Omega_{1}}\left(\widetilde{\Omega_{1}}\right),
$$

where $\Delta$ is any disc contained in $D(0 ; \delta)$

Let $\mathrm{j}: \Omega_{1} \hookrightarrow \widetilde{\Omega_{1}}$ be the canonical holomorphic imbedding of $\Omega_{1}$ into $\widetilde{\Omega_{1}}$. As before, there exist holomorphic mappings

$$
\widetilde{\mathcal{D}_{r}}(\cdot ; \eta): D(0 ; r) \rightarrow \widetilde{\Omega_{1}} \forall r \in(0,1), \text { and } \widetilde{\psi(}(; \eta): D \rightarrow \widetilde{\Omega_{1}}
$$

such that

a) For each $\eta$ with $|\eta|<\delta$ :

$$
\begin{aligned}
\pi^{\Omega_{1}} \circ \widetilde{\mathcal{D}_{r}}(\zeta ; \eta) & =\left(\zeta, \mathcal{D}_{r}^{(\eta)}(\zeta)\right) \forall \zeta \in D(0 ; r) \text { when } r \in(0,1), \text { and } \\
\pi^{\Omega_{1}} \circ \widetilde{\psi}(\zeta ; \eta) & =(\zeta, \psi(\zeta)+\eta) \forall \zeta \in D .
\end{aligned}
$$

b) $\mathrm{j}\left(\zeta, \mathcal{D}_{r}^{(\eta)}(\zeta)\right)=\widetilde{\mathcal{D}_{r}}(\zeta ; \eta)$ wherever the left-hand side is defined, and $\forall r \in(0,1)$.

c) $\mathrm{j}(\zeta, \psi(\zeta)+\eta)=\widetilde{\psi}(\zeta ; \eta)$ wherever the left-hand side is defined.

Arguing exactly as in the proof of Theorem 1.1. we can find a disc $\Delta_{*} \subset D(0 ; \delta)$, centered at the origin, and an appropriate open set $\omega_{2}$ satisfying $\overline{\Omega_{2}} \subset \omega_{2} \subset \Omega_{1}$, such that if we define

$$
\tilde{\mathrm{j}}(z, w):= \begin{cases}\widetilde{\psi}(z ; w-\psi(z)), & \text { if }(z, w) \in U\left(\Delta_{*}\right), \\ \mathrm{j}(z, w), & \text { if }(z, w) \in \omega_{2},\end{cases}
$$

(where $U\left(\Delta_{*}\right)$ is as defined by (3.3) above), then $\tilde{j}$ is holomorphic and well defined. Holomorphicity follows from (a) above, while (c) implies that

$$
\widetilde{\psi}(z ; w-\psi(z))=\mathrm{j}(z, \psi(z)+\{w-\psi(z)\})=\mathrm{j}(z, w) \quad \forall(z, w) \in U\left(\Delta_{*}\right) \cap \omega_{2} .
$$


We define $\Omega^{*}:=U\left(\Delta_{*}\right) \cup \omega_{2}$. As before, given any $f \in \mathcal{O}\left(\Omega_{1}\right)$, we define $\tilde{f} \in \mathcal{O}\left(\Omega_{*}\right)$ by $\tilde{f}:=\mathcal{E}(f) \circ \tilde{\mathrm{j}}$. In view of (3.4), $\left.\left.\widetilde{f}\right|_{\omega_{2}} \equiv f\right|_{\omega_{2}}$. Since $\Omega_{*}$ is a neighbourhood of $\Gamma(\psi) \cup(\partial D \times D)$, i.e. is a classical Hartogs configuration, $\tilde{f}$ has a holomorphic extension to $D \times D$, whence $\left.f\right|_{\Omega_{2}}$ has a holomorphic extension to $D \times D$. This concludes our proof.

3.3. The proof of Theorem 1.3. The proof of Theorem 1.3 proceeds along the same lines as the proof of the first theorem. The essential difference is that we find an $\varepsilon>0$ such that $F(D)+\eta \subset$ $\Omega \forall \eta \in D(0 ; 2 \varepsilon)^{m}$, and then apply Lemma 2.5 to the pairs $\left(F_{1}, \varepsilon\right), \ldots,\left(F_{m}, \varepsilon\right)$ to obtain a map $G=\left(G_{1}, \ldots, G_{m}\right)$ all of whose components obey the conclusions of Lemma 2.5 Let us write $G_{j}\left(r e^{i \theta}\right)=\sum_{n=-N(j)}^{N(j)} B_{n}(r) e^{i n \theta}, j=1,2, \ldots, m$. We apply Lemma 2.1 by defining

$$
b_{n}(r):=B_{j n}(r), \quad j \neq 0, \quad b_{0}(r):=B_{j 0}(r)+\eta,
$$

for each $j=1, \ldots, m$, and obtain

$$
\begin{gathered}
\mathcal{A}_{r}^{(\eta)}:=\left(\mathcal{A}_{1, r}^{(\eta)}, \ldots, \mathcal{A}_{m, r}^{(\eta)}\right): \overline{\operatorname{Ann}}(0 ; r, 1) \rightarrow D^{m}, \\
\mathcal{A}_{r}^{(\eta)} \in \mathcal{O}[\operatorname{Ann}(0 ; r, 1)] \cap \mathcal{C}\left[\overline{\operatorname{Ann}}(0 ; r, 1) ; D^{m}\right] \text { for every } \eta \in D(0 ; \delta)^{m} \text { and } \forall r \in(0 ; 1),
\end{gathered}
$$

where $\delta>0$ is chosen to be so small that:

- $\sup _{\partial D}\left|H_{j}\right|+\delta<1$ for $j=1, \ldots, m$;

- $\delta+\sum_{n=-N}^{N}\left|B_{j n}(r)\right| / r^{n}<1 \forall r \in(0 ; 1]$ and $j=1, \ldots, m$; and

- $H(D)+\eta \subset \Omega_{1} \forall \eta \in D(0 ; \delta)^{m}$.

As before, there exists a $D^{m}$-valued function $\psi:=\left(\psi_{1}, \ldots, \psi_{m}\right) \in \mathcal{O}(D) \cap \mathcal{C}\left(\bar{D} ; D^{m}\right)$ such that for each $\eta \in D(0 ; \delta)^{m}$

$$
\lim _{r \rightarrow 0^{+}} \mathcal{A}_{r}^{(\eta)}\left(\zeta_{0}\right)=\psi\left(\zeta_{0}\right)+\eta \text { for each fixed } \zeta_{0} \in D \backslash\{0\} .
$$

Defining

$$
\begin{aligned}
H^{(\eta)} & :=\left(H_{1}(\zeta)+\eta_{1}, \ldots, H_{n}(\zeta)+\eta_{m}\right) \quad \forall \eta=\left(\eta_{1}, \ldots, \eta_{m}\right) \in D(0 ; \delta)^{m}, \\
\mathfrak{K}^{(\eta)} & :=\Gamma(\psi+\eta) \cup\left[\cup_{0<r<1} \Gamma\left(\mathcal{A}_{r}^{(\eta)}\right)\right] \backslash \Omega_{1},
\end{aligned}
$$

we see that properties (1)-(3) in the proof of Theorem 1.1 hold for $\left\{\mathcal{A}_{r}^{(\eta)}\right\}_{r \in(0,1)}$ and $\psi$ in our new context - the only difference being that the relevant functions are vector-valued, and $\eta$ varies in a polydisc $D(0 ; \delta)^{m}$. Therefore, $\left\{\Gamma\left(\mathcal{A}_{r}^{(\eta)}\right)\right\}_{r \in(0,1)}$ is a continuous family of analytic annuli attached to $\Gamma\left(H^{(\eta)}\right) \cup\left(\partial D \times D^{m}\right)$, which accumulate onto $\Gamma(\psi+\eta)$ as $r \rightarrow 0^{+}$. The analytic annuli $\Gamma\left(\mathcal{A}_{r}^{(\eta)}\right)$ with $r \approx 1$ are contained in $\Omega_{1}$. As before, the Kontinuitätssatz tells us that

$$
U(\mathbf{P}):=\bigcup_{\eta \in \mathbf{P}} \Gamma(\psi+\eta) \subset \pi^{\Omega_{1}}\left(\widetilde{\Omega}_{1}\right),
$$

where $\mathbf{P}$ is any polydisc contained in $D(0 ; \delta)^{m}$.

Arguing exactly as before, we can find a sufficiently small polydisc $\mathbf{P}_{*} \subset D(0 ; \delta)^{m}$ centered at the origin, an appropriately chosen domain $\omega_{2}$ such that $\overline{\Omega_{2}} \subset \omega_{2} \subset \Omega_{1}$, and a mapping $\tilde{\mathrm{j}}: U\left(\mathbf{P}_{*}\right) \cup \omega_{2}$ (here, $U\left(\mathbf{P}_{*}\right)$ is as defined in (3.5) above) such that

- $\tilde{\mathrm{j}} \in \mathcal{O}\left(U\left(\mathbf{P}_{*}\right) \cup \omega_{2}\right)$, and

- $\left.\left.\widetilde{j}\right|_{\omega_{2}} \equiv \mathrm{j}\right|_{\omega_{2}}$.

We use this mapping $\tilde{j}$ exactly as in the previous two theorems to complete this proof. 


\section{ExAmples}

We begin by showing that one can construct a Hartogs-type configuration $\Gamma(F) \cup(\partial D \times D)$ such that $\sup _{\bar{D}}|F|$ is as large as we want and such that functions holomorphic in any small neighbourhood of this configuration extend to $D \times D$ in the manner described in Theorem 1.1 A related counterexample to this sort of a phenomenon is the case when $\Gamma(F)$ is a Wermer disc (see Example 4.2 below for a definition). In that case, there is no analytic continuation, provided $\sup _{\bar{D}}|F|$ is sufficiently large. We explain under the heading Example 4.2 why this does not contradict Theorem 1.1

Theorem [1.3 is not true if $F$ occuring therein is replaced by an arbitrary smooth, $D^{m}$-valued function. This is the content of Rosay's counterexample in [8]. Under Example 4.3] below, we discuss how Rosay's counterexample fails to meet the hypotheses of Theorem 1.3

We begin with our first example.

Example 4.1. An example showing that given any $N \in \mathbb{N}$, there is a $F \in \mathcal{C}^{\infty}(\bar{D} ; \mathbb{C})$ such that $\sup _{\bar{D}}|F|>N, \sup _{\partial D}|F|<1$, and such that small connected neighbourhoods of $\Gamma(F) \cup(\partial D \times D)$ exhibit the analytic-continuation phenomenon described in Theorem 1.1.

We are given $N \in \mathbb{N}$. Let $\chi \in \mathcal{C}^{\infty}([0, \infty) ;[0,1))$ be a smooth cut-off on $[0, \infty)$ such that

$$
\begin{aligned}
\left.\chi\right|_{[1 /(N+1), \infty)} & \equiv \frac{N+(1 / 2)}{N+1}, \\
\chi & \equiv 0 \text { in a relatively open neighbourhood of } 0 .
\end{aligned}
$$

We define

$$
F\left(r e^{i \theta}\right):=\frac{\chi(r)}{r} e^{-i \theta}
$$

Clearly

$$
\begin{aligned}
\left|F\left(e^{i \theta} /(N+1)\right)\right| & =N+(1 / 2)>N \\
|F(\zeta)| & =\frac{N+(1 / 2)}{N+1}<1 \quad \forall \zeta \in \partial D \\
r\left|A_{-1}(r)\right| & =\chi(r) \leq \frac{N+(1 / 2)}{N+1}<1 \quad \forall r \in(0,1] .
\end{aligned}
$$

These conditions imply that for any connected neighbourhood $\Omega_{1} \supset \Gamma(F) \cup(\partial D \times D)$, any connected open set $\Omega_{2}$ satisfying $(\partial D \times D) \subset \overline{\Omega_{2}} \subset \Omega_{1} \cap(\{|z| \geq 1\} \times D)$, and for any $f \in \mathcal{O}\left(\Omega_{1}\right)$, $\left.f\right|_{\Omega_{2}}$ extends holomorphically to $D \times D$.

Wermer presents an example of a function $g \in \mathcal{C}^{\infty}(\bar{D})$ [6] with the property that $\Gamma(g)$ is totally real, but $\left.g\right|_{\partial D} \equiv 0$. This allows us to define a function $F=M g$ - where $M>0$ is sufficiently large - such that the configuration $\Gamma(F) \cup(\partial D \times D)$ resists analytic continuation of the type described in Theorem 1.1 We now explain how the relevant $F$ fails to satisfy the hypothesis of Theorem 1.1 
Example 4.2. Wermer's disc.

The graph of the function

$$
g(z, \bar{z})=\bar{z}\left(1-|z|^{4}\right)+i \bar{z}\left(1-|z|^{2}\right)
$$

is a totally-real surface in $\mathbb{C}^{2}$. This follows from an easy computation; see details in [6. Example 6.1]. Therefore, the domain

$$
\mathcal{D}_{\delta}:=\left\{(z, w) \in \mathbb{C}^{2}:|z|<1+\delta,|w-g(z)|<\delta\right\}
$$

is a pseudoconvex domain for all $\delta>0$ sufficiently small. Notice that $\mathcal{D}_{\delta} \supset \partial D \times D(0, \delta)$. Let $\delta^{*}>0$ be so small that $D \times\{0\} \varsubsetneqq \mathcal{D}_{\delta^{*}}$ and $\mathcal{D}_{\delta^{*}}$ is pseudoconvex. Then, for each domain $\Omega_{1} \supset \Gamma(g) \cup\left(\partial D \times D\left(0 ; \delta^{*}\right)\right)$ such that $\Omega_{1} \subset \mathcal{D}_{\delta^{*}}$, there would exist a function $f \in \mathcal{O}\left(\Omega_{1}\right)$ such that $f$ does not extend holomorphically to the bidisc $D \times D\left(0 ; \delta^{*}\right)$, because $\mathcal{D}_{\delta^{*}}$ is a domain of holomorphy but does not contain $D \times\{0\}$. We now define

$$
F(z):=\frac{1}{\delta^{*}} g(z), \widetilde{\mathcal{D}_{\delta^{*}}}:=\left\{(z, w) \mid\left(z, \delta^{*} w\right) \in \mathcal{D}_{\delta^{*}}\right\} .
$$

By construction, $\widetilde{\mathcal{D}_{\delta^{*}}}$ is a pseudoconvex domain that contains $\Gamma(F) \cup(\partial D \times D)$ but does not contain $D \times\{0\}$. By our preceding remarks, the Hartogs-Chirka type configuration just constructed does not admit analytic continuation in the manner described in Theorem 1.1

Notice that $F\left(r e^{i \theta}\right)=A_{-1}(r) e^{-i \theta}$, where

$$
A_{-1}(r)=\frac{r}{\delta^{*}}\left\{\left(1-r^{4}\right)+i\left(1-r^{2}\right)\right\} .
$$

We will now show that

$$
r\left|A_{-1}(r)\right|=\frac{r^{2}}{\delta^{*}}\left\{\left(1-r^{4}\right)^{2}+\left(1-r^{2}\right)^{2}\right\}^{1 / 2} \gg 1 \text { for some } r \in(0,1],
$$

whence Theorem 1.1 is inapplicable to the above configuration. For this purpose, we will need an upper bound for the quantity $\delta^{*}$ introduced above, and we make the following

Claim : $\delta^{*}<0.0061$. To see this, we refer to the Berndtsson-Słodkowski inequality - see [1, Prop.2.3/(b)] - determining when a surface of the form

$$
\mathcal{S}=\left\{(z, w) \in \Omega \times \mathbb{C}|| w-G(z) \mid=e^{-u(z)}\right\},
$$

(here $\Omega$ is a domain in $\mathbb{C}, G$ and $u$ are smooth functions, and $u$ is real-valued) is pseudoconvex. The desired inequality is

$$
\mathcal{S} \text { is pseudoconvex } \Longleftrightarrow-u_{z \bar{z}} \leq e^{2 u}\left|G_{\bar{z}}\right|^{2}-e^{u}\left|G_{z \bar{z}}+2 u_{z} G_{\bar{z}}\right| .
$$

For $\mathcal{D}_{\delta^{*}}$ to be pseudoconvex, we require that the surface $\mathcal{S}_{\delta^{*}}:=\left\{(z, w) \in D \times \mathbb{C}|| w-g(z) \mid=\delta^{*}\right\}$ be pseudoconvex. Applying (4.1) to the surface $\mathcal{S}_{\delta^{*}}$ yields the following restriction on $\delta^{*}$.

$$
0<\delta^{*} \leq \frac{\left(1-3|z|^{4}\right)^{2}+\left(1-2|z|^{2}\right)^{2}}{|z| \sqrt{36|z|^{4}+4}} \quad \forall|z| \leq 1
$$

In other words,

$$
0<\delta^{*} \leq \min _{r \in[0,1]} \frac{\left(1-3 r^{4}\right)^{2}+\left(1-2 r^{2}\right)^{2}}{r \sqrt{36 r^{4}+4}}
$$

and one can use any computational software package to show that the right-hand side of the above inequality is greater than 0.0061 . Hence the claim. 
One can also compute that $\max _{r \in[0,1]} r^{2}\left\{\left(1-r^{4}\right)^{2}+\left(1-r^{2}\right)^{2}\right\}^{1 / 2} \approx 0.456$. Thus

$$
\max _{r \in[0,1]} r\left|A_{-1}(r)\right|>\frac{0.456}{0.0061} \gg 1,
$$

which violates condition (1.1).

Example 4.3. Rosay's counterexample.

Rosay shows that one can find an arbitrarily small, strictly pseudoconvex neighbourhood $\Omega$ of $\partial D \times D^{2}$ and a $D^{2}$-valued function $F$ such that $\Gamma(F) \cup\left(\partial D \times D^{2}\right)$ is holomorphically convex. Specifically

$$
\begin{aligned}
\Omega:=\left\{\left(z_{1}, z_{2}, z_{3}\right) \in \mathbb{C}^{3}:\left\{\left(\left|z_{1}\right|^{2}-1\right)^{2}+\right.\right. & \left.s_{1}\left|z_{2}\right|^{2}\right\}^{N}+\left|\frac{z_{3}}{N}\right|^{2 N} \\
& \left.+\alpha\left\{\left(\left|z_{1}\right|^{2}-1\right)^{2}+s_{1}\left|z_{2}\right|^{2}+\left|z_{3}\right|^{2}\right\}<s^{2 N}+\alpha s^{2}\right\},
\end{aligned}
$$

where

- $s>0$ is small, and $s_{1}=s(1-\delta)<s$ for a fixed, small $\delta>0$, and

- One first chooses $N$ large enough that $s_{1}^{2 N}+1 / N^{2 N}<s^{2 N}$, and then chooses $\alpha$ sufficiently small so as to ensure that $\partial D \times D^{2} \Subset \Omega$.

Write $F=\left(F_{1}, F_{2}\right)$. In Rosay's construction

$$
F_{1}\left(r e^{i \theta}\right):=\kappa \chi(r) e^{i \theta}
$$

where $\chi \in \mathcal{C}^{\infty}[0,1]$ with $0 \leq \chi \leq 1$, such that $\chi \equiv 1$ off a small relative neighbourhood of $0 \in[0,1]$ and $\chi \equiv 0$ on a smaller neighbourhood of 0 . The quantity $\kappa$ will be described presently. The function $F_{2}$ is required to be identically zero in an open set contained in $\left\{r e^{i \theta}: r \in \operatorname{supp}(\chi)\right\}$, and to satisfy $\partial F_{1} / \partial \bar{z}_{1} \neq 0$ wherever $F_{1} \equiv 0$. Therefore, $F_{2}$ will have negative Fourier modes.

Our interest is in examining $F_{1}$. The constant $\kappa$ is so chosen that

$$
\Gamma(F) \cap \bar{\Omega}=\left\{\left(R e^{i \theta}, \kappa e^{i \theta}, 0\right): \theta \in[0,2 \pi)\right\},
$$

and such that $\Gamma(F) \cap \bar{\Omega}$ is a complex-tangential curve in the surface $\partial \Omega \cap\left\{z_{3}=0\right\}$. Write $z_{j}:=x_{j}+i y_{j}, j=1,2$. It is easy to determine what the magnitudes of $\kappa$ and $R$ (which is close to $1)$ should be by visualizing $\omega:=\bar{\Omega} \cap\left(\mathbb{R}^{2} \times\{0\}\right)$. Then $(R, \kappa)$ are the coordinates of the point of tangency, in the first quadrant, of the line through the origin that is tangential to $\partial \omega$ (then, the complex span of this line contains the tangent line to the curve $\Gamma(F) \cap \bar{\Omega})$. By construction, the point $\left(1, s / s_{1}\right) \in \partial \omega$ lies below the line just described, whence the line $x_{1}=x_{2}$ lies below this line, in the first quadrant. Thus, in the notation of Theorem 1.3

$$
\kappa / R:=A_{11}(R) / R>1,
$$

whence, by construction

$$
A_{11}(r) / r>1 \quad \forall r \in \chi^{-1}\{1\} .
$$

This violates the condition (1.2). 


\section{REFERENCES}

[1] B. Berndtsson, Levi-flat surfaces with circular sections, Several Complex Variables, Math. Notes (John Erik Fornaess ed.), Princeton University Press, Princeton, NJ (1993), pp. 136-159.

[2] G. Bharali, Some generalizations of Chirka's extension theorem, Proc. Amer. Math. Soc. 129 (2001), 3665-3669.

[3] E.M. Chirka, Generalized Hartogs' lemma and non-linear $\bar{\partial}$-equation, Complex Analysis in Contemporary Mathematics (E.M. Chirka, ed.), FAZIS, Moscow (in Russian) (2002).

[4] E.M. Chirka and J.-P. Rosay, Remarks on the proof of a generalized Hartogs lemma, Ann. Pol. Math. 70 (1998), 43-47.

[5] E.M. Chirka and E.L. Stout, A Kontinuitätssatz, Topics in Complex Analysis (P. Jacóbczak, W. Pleśniak, eds.), Banach Center Publications, Warsaw, 31 (1995), pp. 143-150.

[6] L. Hörmander and J. Wermer, Uniform approximation on compact sets in $\mathbb{C}^{n}$, Math. Scand. 23 (1968), 5-21.

[7] S.M. Ivashkovich and V.V. Shevchishin, Deformations of noncompact complex curves, and envelopes of meromorphy of spheres (in Russian), Mat. Sb. 189 (1998), 23-60.

[8] J.-P. Rosay, A counterexample related to Hartogs' phenomenon (A question by E. Chirka), Michigan Math. J. 45 (1998), 529-535.

Department of Mathematics, University of Michigan, 525 East University Ann Arbor, Mi 48109

E-mail address: barrett@umich.edu

E-mail address: bharali@umich.edu 